\title{
Taking the vector vortex coronagraph to the next level for ground- and space-based exoplanet imaging instruments: review of technology developments in the USA, Japan, and Europe
}

Dimitri Mawet $^{a, b}$, Naoshi Murakami ${ }^{c}$, Christian Delacroix $^{d}$, Eugene Serabyn $^{b}$, Olivier Absil ${ }^{d}$, Naoshi Baba ${ }^{c}$, Jacques Baudrand ${ }^{e}$, Anthony Boccaletti ${ }^{e}$, Rick Burruss ${ }^{b}$, Russell Chipman ${ }^{f}$, Pontus Forsberg $^{g}$, Serge Habraken ${ }^{d}$, Shoki Hamaguchi ${ }^{c}$, Charles Hanot ${ }^{d}$, Akitoshi Ise ${ }^{c}$, Mikael Karlsson $^{g}$, Brian Kern ${ }^{b}$, John Krist ${ }^{b}$, Andreas Kuhnert ${ }^{b}$, Marie Levine ${ }^{b}$, Kurt Liewer ${ }^{b}$, Stephen

McClain $^{f}$, Scott McEldowney ${ }^{h}$, Bertrand Mennesson ${ }^{b}$, Dwight Moody ${ }^{b}$, Hiroshi Murakami ${ }^{i}$, Albert Niessner $^{b}$ Jun Nishikawa $^{j}$, Nada O’Brien ${ }^{k}$, Kazuhiko Oka ${ }^{c}$, Peggy Park ${ }^{b}$, Pierre Piron ${ }^{d}$, Laurent Pueyo ${ }^{l}$, Pierre Riaud ${ }^{d}$, Moritsugu Sakamoto ${ }^{c}$, Motohide Tamura $^{j}$, John $_{\text {Trauger }}{ }$,

David Shemo $^{k}$, Jean Surdej ${ }^{d}$, Nelson Tabiryan ${ }^{m}$, Wesley Traub ${ }^{b}$, James Wallace ${ }^{b}$, Kaito Yokochi $^{n}$

${ }^{a}$ European Southern Observatory, Alonso de Córdova 3107, Vitacura 7630355, Santiago, Chile;

${ }^{b}$ Jet Propulsion Laboratory - California Institute of Technology, 4800 Oak Grove Dr, Pasadena, CA 91109, USA;

${ }^{c}$ Hokkaido University, Sapporo, Hokkaido 060-8628, Japan;

${ }^{d}$ University of Liège, 17 Allée du 6 Août, 4000 Sart Tilman, Belgium;

${ }^{e}$ LESIA, Observatoire de Paris, 5 pl. J. Janssen, F-92195 Meudon, France;

fUniversity of Arizona, 1630 E University Blvd., Tucson, AZ 85721, USA ;

${ }^{g}$ Uppsala University, Lägerhyddsvägen 1, P.O. Box 534, SE-751 21 Uppsala, Sweden;

${ }^{h}$ Microsoft, 1 Microsoft Way, Redmond, WA 98052, USA;

${ }^{i}$ Japan Aerospace Exploration Agency, 3-1-1 Yoshinodai, Chuo-ku, Sagamihara, Kanagawa 252-5210, Japan;

${ }^{j}$ National Astronomical Observatory of Japan, 2-21-1 Osawa, Mitaka, Tokyo 181-8588, Japan;

${ }^{k}$ JDSU, 2789 Northpoint Parkway, Santa Rosa, CA 95407, USA;

${ }^{l}$ Space Telescope Science Institute, 3700 San Martin Drive, Baltimore, MD 21218, USA; ${ }^{m}$ BEAM Engineering for Advanced Measurements Co., 809 S. Orlando Ave., Suite I, Winter Park, FL 32789, USA ${ }^{n}$ Tokyo University of Agriculture and Technology, Graduate School of Engineering, Tokyo 184-8588, Japan

\begin{abstract}
The Vector Vortex Coronagraph (VVC) is one of the most attractive new-generation coronagraphs for ground- and space-based exoplanet imaging/characterization instruments, as recently demonstrated on sky at Palomar and in the laboratory at JPL, and Hokkaido University. Manufacturing technologies for devices covering wavelength ranges from the optical to the mid-infrared, have been maturing quickly. We will review the current status of technology developments supported by NASA in the USA (Jet Propulsion Laboratory-California Institute of Technology, University of Arizona, JDSU and BEAMCo), Europe (University of Liège, Observatoire de ParisMeudon, University of Uppsala) and Japan (Hokkaido University, and Photonics Lattice Inc.), using liquid crystal polymers, subwavelength gratings, and photonics crystals, respectively. We will then browse concrete perspectives for the use of the VVC on upcoming ground-based facilities with or without (extreme) adaptive optics, extremely large ground-based telescopes, and space-based internal coronagraphs.
\end{abstract}

Further author information: send correspondence to dmawet@eso.org

Techniques and Instrumentation for Detection of Exoplanets $\mathrm{V}$, edited by Stuart Shaklan,

Proc. of SPIE Vol. 8151, 815108 • @ 2011 SPIE · CCC code: 0277-786X/11/\$18 · doi: 10.1117/12.896059

Proc. of SPIE Vol. $8151815108-1$ 
Keywords: High contrast imaging, exoplanets, phase-mask coronagraphy, optical vortex, polarization, liquid crystal polymers, photonics crystals, subwavelength gratings

\section{INTRODUCTION}

The Vector Vortex Coronagraph (VVC) is one of the most recent members of the phase-mask coronagraph family. Coronagraphy, named after the (almost) century-old invention of Bernard Lyot to observe the Solar corona independently of natural eclipses, is nowadays a generic term qualifying techniques used to enhance contrast in astrophysical scenes where the goal is imaging faint features near bright objects. The typical example is an extrasolar planet located less than an arcsecond from its much brighter host star. The vortex coronagraph, or $\mathrm{VC},{ }^{1-4}$ can observe very close to bright stars, like most of its siblings of the phase-mask coronagraph family. For that and many other reasons such as high throughput, clear off-axis discovery space and simplicity, all detailed previously e.g. in Ref. $5-7$, it is one of the most promising of coronagraph types.

The $\mathrm{VVC}^{1,8}$ is one possible and attractive realization of the vortex coronagraph based on a circularly symmetric halfwave plate (HWP). HWP are common optical devices made out of natural or artificial birefringent media, used to manipulate the polarization state of light. The nominal textbook effect of a HWP on linearly polarized light is to rotate it by $-2 \alpha$, where $\alpha$ is the angle between the input polarization vector and the fast axis of the HWP. It is worth noting that the effect of a HWP on circularly polarized light is to revert its handedness (left-circular becomes right-circular and vice-versa), while inducing a notable phase shift equal to the same $-2 \alpha$. Indeed, the definition of circular polarization is nothing more than a linear polarization rotating at the angular frequency $\omega$, equal to the pulsation of the electromagnetic field.

The difficulty of mastering the manufacturing of the VVC comes from the necessity of manipulating the polarization vector in a space-variant manner, i.e. it needs to be dramatically modulated across spatial scales of less than a mm, with precisions of a few microns and fractions of a degree. In this paper, after reviewing the physics behind the VC and VVC, we will review the three main technological approaches currently used to manufacture the VVC. In the US, the liquid crystal polymer (LCP) approach is being pursued. In Europe, the main route being followed is that of subwavelength gratings, even though LCPs are also currently explored (note that one of the main sources of raw LCP material is produced by Rolic Inc. in Switzerland). Finally, in Japan, the selected technology is photonics crystals. The reason for the geographical differences seems to be linked to the local availability of the respective technologies in laboratories or private companies. Each one of these technological choices has advantages and drawbacks. However, we will show here that, instead of overlapping each other, these developments cover different parts of the field of application spectrum, conveniently complementing each other.

\section{VORTEX CORONAGRAPH PRINCIPLE}

A vortex coronagraph applies a helical phase of the form $e^{i \phi}$, with $\phi=l \theta$, where $\theta$ is the focal plane azimuthal coordinate and $l$ the (even) vortex "topological charge", to a telescope's focal plane field, by means of a transparent phase mask (Fig. 1). The light then propagates downstream to a pupil image (the "Lyot" plane), where for an ideal unobscured circular input pupil (Fig. 1, left), all of the (on-axis) starlight appears outside of the geometric image of the input pupil, rendering the interior of the output pupil empty of the incoming on-axis illumination (Fig. 1, right). A Lyot-plane aperture (the Lyot stop) then blocks all of the diffracted starlight. Light from off-axis objects misses the center of the vortex and propagates normally.

The effect of a topological charge $l=2$ vortex phase, $e^{i 2 \theta}$, applied to the ideal focal plane field (Airy pattern), $\frac{2 J_{1}(k \rho A)}{k \rho A}$, of a filled circular aperture of radius $A$, where $\mathrm{k}$ is the wavenumber and $\rho$ is the radial coordinate in the focal plane, has been calculated analytically, ${ }^{1,3}$ and the Fourier transform of $e^{i 2 \theta} \times \frac{2 J_{1}(k \rho A)}{k \rho A}$ to the pupil (Lyot) plane yields

$$
E_{\text {Lyot }}(r, \psi)= \begin{cases}0 & r<A \\ e^{i 2 \psi}\left(\frac{A}{r}\right)^{2} & r>A\end{cases}
$$

The field inside the pupil area is null, while outside the pupil area it has a $1 / r^{2}$ falloff. The perfect cancellation holds true for any even (non-zero) value of the topological charge, as shown in Refs. 1,3.

Proc. of SPIE Vol. $8151815108-2$ 


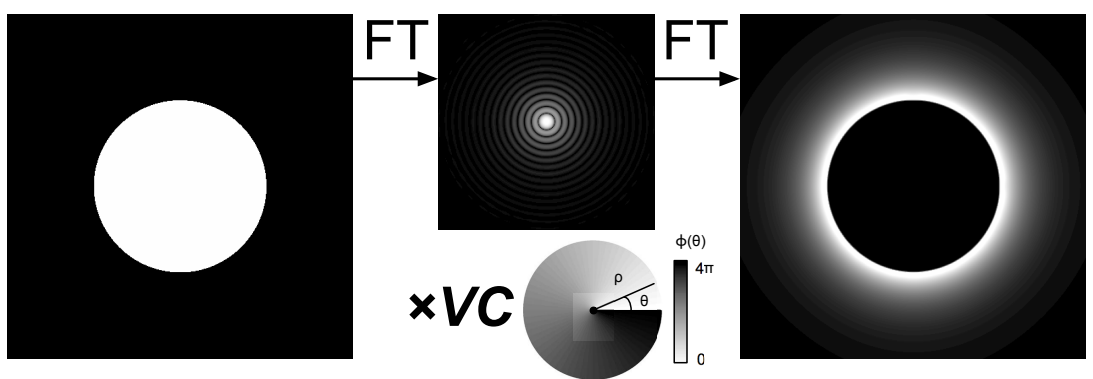

Figure 1. Illustration of the diffraction effect of the vortex phase mask on a filled aperture (left). All of the on-axis coherent light appears outside of the geometric image of the input pupil (right). A circular aperture (Lyot stop) then blocks it all. FT stands for Fourier transform.

\section{A. Linear Polarization $\quad$ B. Circular Polarization}

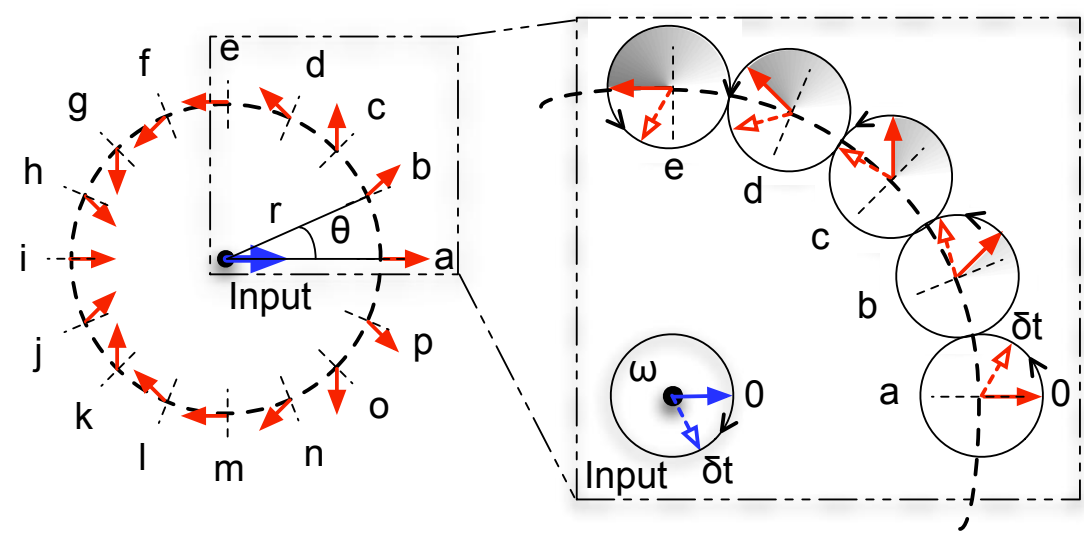

Figure 2. The VVC azimuthal phase ramp. Panel A: rotationally symmetric HWP with an optical axis orientation that rotates about the center (dashed lines perpendicular to the circumference). The net effect of a HWP on a linear impinging polarization is to rotate it by $-2 \times \alpha$ where $\alpha$ is the angle between the incoming polarization direction and the fast optical axis. An incoming horizontal polarization (blue arrow) is transformed by the vector vortex so that it spins around its center twice as fast as the azimuthal coordinate $\theta$ (red arrows). Panel B: for circular polarization, the output field rotation is strictly equivalent to a phase delay (the starting angle 0 is rotated; therefore phase delayed). The angle of local rotation of the polarization vector corresponds to a "geometrical" phase: upon a complete rotation about the center of the rotationally symmetric HWP, it has undergone a total $2 \times 2 \pi$ phase ramp, which corresponds to the definition of an optical vortex of topological charge 2 .

\section{PHYSICAL PROPERTIES OF THE VECTOR VORTEX}

As noted above, the net effect of a HWP on circularly polarized light is to change its handedness and apply a phase delay proportional to $-2 \alpha$, where $\alpha$ is the angle between the instantaneous input rotating polarization vector and the fast axis of the HWP. This additional delay is the essence of the so-called "geometrical" or PancharatnamBerry phase. ${ }^{9,10}$ Generating a phase ramp with HWP is thus straightforward in theory: one just needs to make a circularly symmetric HWP. Of course, it is easier said than done. The best known and readily available HWP are made out of birefringent crystals. Tailoring the fast axis orientation is almost impossible, at least in a smooth continuous manner, as it would require cutting the crystal and assembling the pieces together very precisely. Note that this technique has recently been used to manufacture achromatic four-quadrant phase-masks $(\mathrm{FQPM})^{11,12}$ for SPHERE, ${ }^{13}$ the second-generation Extreme Adaptive Optics (ExAO) planet-finder facility and 3-instrument suite for the Very Large Telescope (VLT). In the particular case of the FQPM, which can be considered as a discrete topological charge 2 vortex, the discretization allowed by the quadrants eases the cutting and assembling process dramatically. Again, that technique would not be practical to generate a smooth phase ramp.

Modern developments in polymer and micro-technologies allowed the development of artificially birefringent media. ${ }^{14,15}$ Birefringence can now be controlled at the molecular level, both in amplitude and direction. It took 


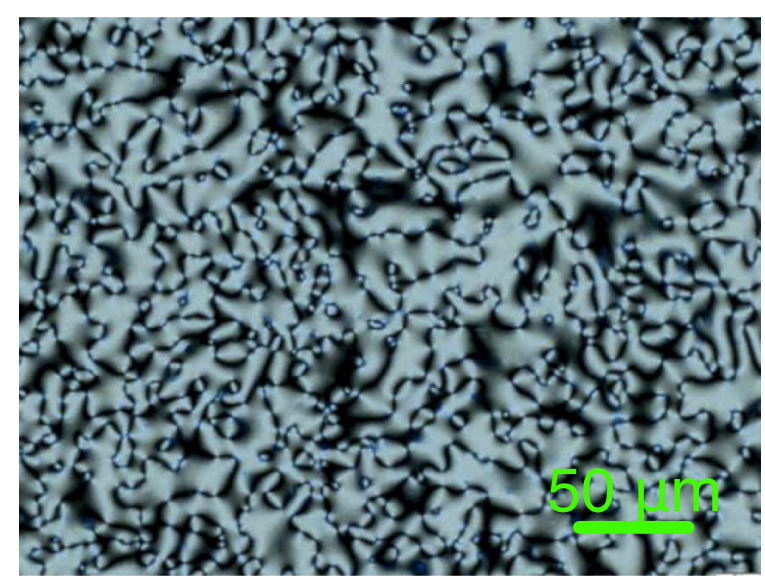

Figure 3. Polarizing microscope picture of raw LCP. Note the natural tendency of LCP to form topological charge 1 vortices at the scale of a few $\mu \mathrm{m}$.

many years between the emergence of these new technologies and their applicability to the making of optical vortices, ${ }^{16}$ but the field is now mature enough to provide competitive devices meeting the toughest specifications. In the following, we will review the 3 main approaches currently being successfully developed.

\section{VVC TECHNOLOGIES}

In this section, we briefly present the 3 technological solutions used to manufacture VVC: liquid crystal polymers, subwavelength gratings, and photonics crystals.

\subsection{Liquid Crystal Polymers}

The liquid crystal phase of a material is defined as the phase between solid state (long range order) and liquid phase (no long range order). Thus, the liquid crystal phase is a state in which the molecules have some average alignment. For example, some liquid crystal polymers have a rod-like molecular structure which is the source of their mechanical and optical anisotropy. In terms of optical properties they exhibit birefringence when the rod axis has a component in the device plane. They also show rigidness along the long axis and have easily polarizable constituents which enable alignment of the liquid crystals.

LCPs are materials that combine the anisotropic properties of liquid crystals with the thermo-mechanical properties of polymers. ${ }^{17,18}$ These materials are usually birefringent materials (Fig. 3). The orientation of the optical axis in the device plane is controlled through the use of an alignment layer. Within a certain temperature range, the liquid crystal precursor will develop the orientation and the positional order characteristics of liquid crystals. Once alignment is established, the liquid crystal precursor can then be cured using standard polymer curing techniques. Once cured, the LCP forms a solid state birefringent material with a permanent alignment. Polymerization of this material is done using a UV curing process. Alignment of liquid crystals in general and LCP specifically, is a key step in making any liquid crystal device. Many techniques are mature and are in use in high volume manufacturing processes today. However, alignment technology is still an area of active research.

\subsection{Subwavelength gratings}

When the period $\Lambda$ of the grating is smaller than the wavelength of the impinging light, it does not diffract as a classical spectroscopic grating. All the incident energy is enforced to propagate only in the zeroth order, the higher orders being diffracted to angles larger than $90^{\circ}$, i.e. within the grating itself. For this reason, subwavelength gratings are also called Zeroth Order Gratings (ZOGs). Whether a diffraction order propagates or not is determined by the well-known grating equation from which a "ZOG condition" on the grating period to wavelength ratio can be derived

$$
\frac{\Lambda}{\lambda} \leq \frac{1}{n_{I} \sin \chi+\max \left(n_{I}, n_{I I I}\right)}
$$



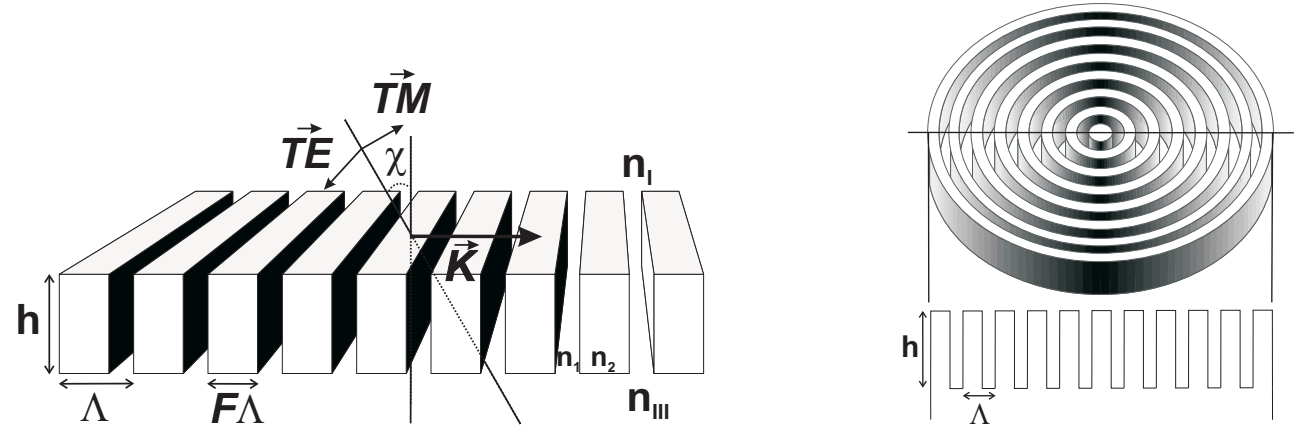

Figure 4. Left: subwavelength grating main parameters: the grating vector $|\vec{K}|=2 \pi / \Lambda$, perpendicular to the grating lines, with $\Lambda$ being the period, the grating depth $h$ and the so-called filling factor $F$, such that $F \Lambda$ is the width of the grating ridges. $\overrightarrow{T E}$ (transverse electric, or $s$ ) and $\overrightarrow{T M}$ (transverse magnetic, or $p$ ) are the polarization components of the incident light. $n_{I}$ and $n_{I I I}$ are the refractive indices of the incident and transmitting media, respectively. $n_{1}$ and $n_{2}$ are the refractive indices of the grating. Right: the VVC of topological charge 2 made out of subwavelength gratings consists in circularly concentric rectangular grooves satisfying the ZOG and HWP conditions. It is also called the Annular Groove Phase Mask, or AGPM.?

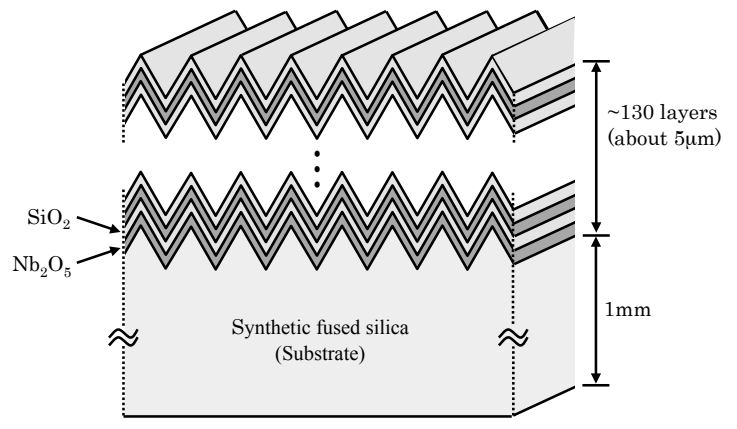

Figure 5. Schematic cross-section of an auto-cloned photonics crystal used to synthesize a VVC. ${ }^{23-25}$

where $\chi$ is the angle of incidence, $n_{I}$ and $n_{I I I}$ are the refractive indices of the incident (superstrate) and transmitting (substrate) media, respectively (see Fig. 4). ZOGs behave like homogeneous media with interesting optical properties, which can be used to e.g. synthesize artificial birefringent achromatic waveplates ${ }^{19,20}$ or monolithic anti-reflective structures. ${ }^{15}$

By carefully controlling the geometry of the grating micro-structure, one can finely adjust the so-called "form birefringence" $\Delta n_{s-p}(\lambda)=n_{s}(\lambda)-n_{p}(\lambda)$, where $n_{s}$ and $n_{p}$ are the two effective indices associated with the subwavelength structure, one for each polarization state: $s$ and $p$. Intuitively, this artificial anisotropy can be interpreted as follows: the incident light sees two different media as its vectorial components vibrate parallel or orthogonal to the grating lines. One can optimize the form birefringence to be proportional to the wavelength in order to compensate for the hyperbolic dependence of the retardance and thus make it quasi-achromatic ${ }^{21}$

$$
\Delta \phi_{s-p}(\lambda)=\frac{2 \pi}{\lambda} h \Delta n_{s-p}(\lambda)
$$

with $h$, the optical path through the birefringent medium. This technique has been successfully used to synthesize achromatic waveplates in the mid-infrared and visible. ${ }^{20,22}$

\subsection{Photonics crystals}

Photonics crystals are articial two-dimensional and three-dimensional periodic structures. In that regard, they can be considered as subwavelength gratings whose periodicity is expanded to the two other dimensions, providing leverage to implement additional optical functions and/or allow different manufacturing processes. 
Table 1. Current characteristics for the 3 main technologies being explored to render the VVC (LCP=liquid crystal polymer, $\mathrm{SG}=$ subwavelength gratings, $\mathrm{PC}=$ photonics crystals). $l$ is the topological charge of the vortex. NIR stands for near-infrared. MIR stands for mid-infrared.

\begin{tabular}{|c|c|c|c|c|}
\hline Technology & Current $\lambda$ coverage & Current $l$ & Vortex center def. & Best contrast \\
\hline $\mathrm{LCP}$ & VIS-NIR & $2-4-\ldots-64$ & $<20 \mu \mathrm{m}\left({ }^{1}\right),<5 \mu \mathrm{m}\left({ }^{2}\right)$ & $\begin{array}{l}3.4 \times 10^{-9} @ 785 \mathrm{~nm}\left(^{a}\right) \\
\sim 2 \times 10^{-8}, 10 \% \mathrm{BW}\left(^{b}\right) \\
\sim 4 \times 10^{-8}, 20 \% \mathrm{BW}\left(^{b}\right)\end{array}$ \\
\hline SG & MIR & 2 & $<5 \mu \mathrm{m}$ & pending \\
\hline $\mathrm{PC}$ & VIS(-NIR) & 2 & $<1 \mu \mathrm{m}$ & $<10^{-6} @ 532 / 633 \mathrm{~nm}\left(^{c}\right)$ \\
\hline
\end{tabular}

(1) JDSU.

$\left({ }^{2}\right)$ BEAMCo.

$\left({ }^{a}\right)$ Without chromatic leakage filtering (Sect. 6), with wavefront control.

$\left({ }^{b}\right)$ With chromatic leakage filtering (Sect. 6), with wavefront control.

${ }^{(c)}$ With chromatic leakage filtering (Sect. 6), without wavefront control.

The particular technology used for synthesizing VVC out of photonics crystals, and the key reason why this technique has been so successful is called "auto-cloning". ${ }^{26,27}$ Auto-cloned photonics crystals consist of patterned multilayer films. It is the only, multi-dimensional, photonics crystal that can be fabricated by conventional sputtering deposition, and established mass production technologies. Fig. 5 shows a typical schematic crosssectional view of an auto-cloned photonics crystal.

\section{STATE OF THE ART}

In this section, we review state of the art for each of the detailed technological approaches.

\subsection{Visible and near-infrared VVC made out of LCP}

These past four years, NASA-JPL has been developing many generations of prototypes and devices in collaboration with JDSU and more recently with BEAMCo. The LCP technology allowed performing breakthrough laboratory demonstrations and rapidly provided usable devices for telescope use. Indeed, two near-infrared topological charge 2 VVC masks have been installed on the 200-inch Hale telescope at Palomar in 2009, and have been used behind an off-axis unobscured subaperture corrected with adaptive optics to successfully image, close binaries, brown dwarfs and extrasolar planets. ${ }^{5,28,29}$

The manufacturing of LCP-VVC, already presented in Ref. 8 uses photo-alignment of a linear polymerizable polymer (LPP) precursor layer (Fig. 6). The LPP layer is spin-coated onto the substrate, baked, and then the alignment is set through exposure to linear polarized UV (LPUV) light. The LPUV light causes the photoalignment layer to simultaneously align and cross-link the long molecular axes. The alignment layer is very thin so its impact on the optical properties of the device is negligeable. The alignment layer is exposed through a
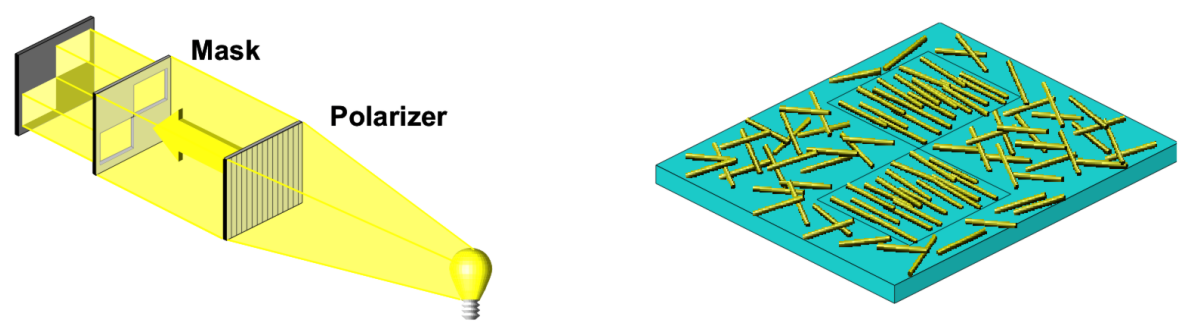

Figure 6. Left. LPUV photoalignment of the LPP layer through an exposure mask. Right: alignment of the LCP rods on the LPUV-aligned precursor LPP layer. Regions with no alignment exhibit a random orientation. 

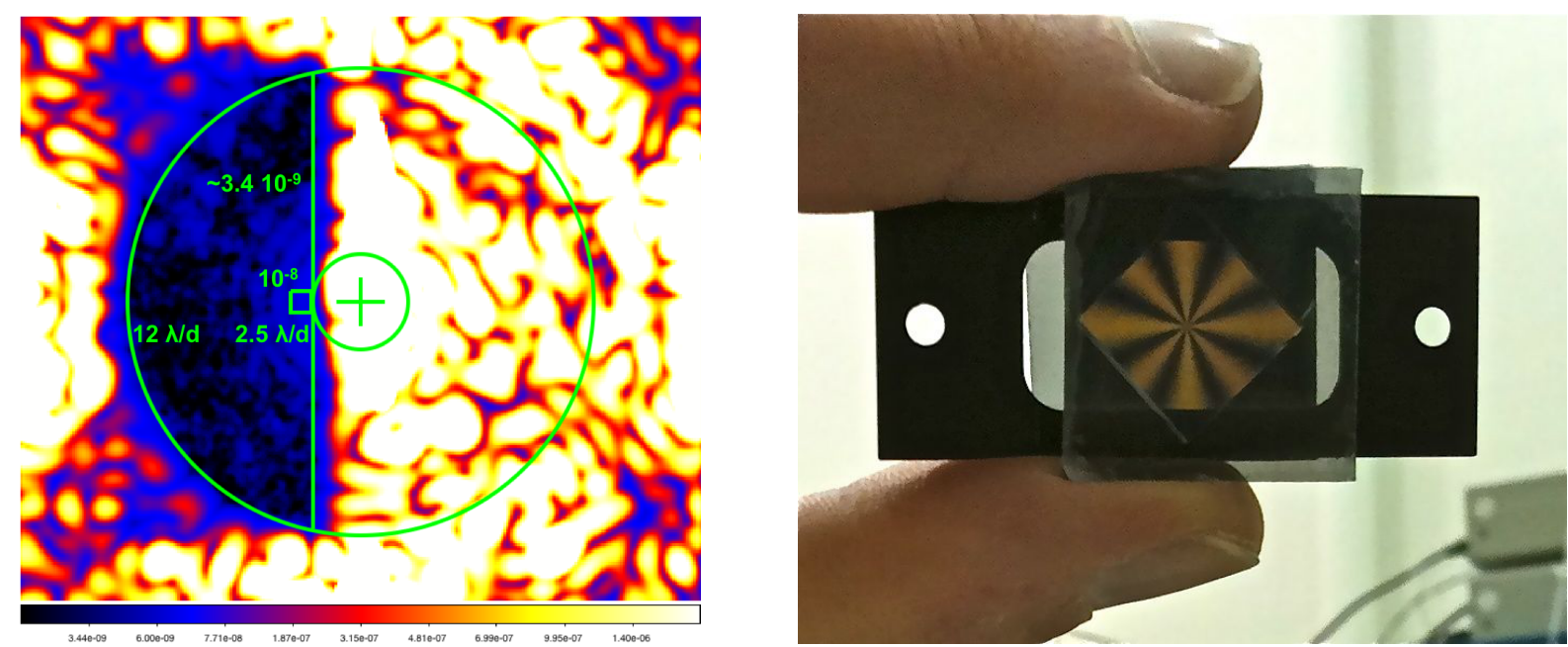

Figure 7. Left: dark hole generated with a VVC of topological charge 4 on the high contrast imaging testbed (HCIT) at JPL using wavefront control. The median contrast between 2.5 and $12 \lambda / d$ is $\sim 3.4 \times 10^{-9}$. Right: picture of the generation-2 VVC of topological charge 4 seen in its mount between crossed polarizers after a run on the HCIT.

narrow wedge shaped aperture located between the substrate and the polarizer. Both the polarizer and the substrate are continuously rotated during the exposure process in order to create a continuous variation in photo-alignment orientation as a function of azimuthal location on the substrate. The variation in fast axis orientation with azimuthal angle is determined by the relative rotation speeds. The LCP is then spin-coated and subsequently polymerized using a UV curing process. It naturally orients itself according to the photo-alignment layer. A post-bake stabilizes the films into a solid VVC.

The LCP technology recently allowed NASA-JPL to test a topological charge 4 VVC demonstrating visible contrasts $3.4 \times 10^{-9}$ (at $785 \mathrm{~nm}$, see Fig. 7$) \sim 2 \times 10^{-8}(10 \%$ bandwidth centered at $800 \mathrm{~nm}), \sim 4 \times 10^{-8}$ ( $20 \%$ bandwidth centered at $800 \mathrm{~nm}$ ) on the high contrast imaging testbed (HCIT). For more details on the test results, see the corresponding communication in these proceedings. ${ }^{30}$ It is worth noting that the most recent developments have shown encouraging results in two areas considered as the two remaining stumbling blocks of the LCP-VVC technology:

1. Intrinsically achromatic 3-layer LCP-VVC (see Sect. 6).

2. Micrometric central region defect: the central region of disorientation is a well-know defect of the LCPVVC. ${ }^{8}$ BEAMCo ${ }^{31}$ has recently tested a new proprietary recording technique showing drastic reduction in the size of the central defect.

Technology developments are ongoing at NASA-JPL, using devices developed and manufactured by JDSU and BEAMCo. The next generation of devices (third generation) is expected within a year. It promises full intrinsic achromatic performance, reduced size of the central defect, and increased reliability.

\subsection{Mid-Infrared VVC made out subwavelength gratings}

The University of Liège and the Observatoire de Meudon have been collaborating with the Angström Laboratory of the Uppsala University to manufacture mid-infrared prototypes with the near-term goal of implementing an L-band prototype in the NAOS-CONICA adaptive optics/infrared camera of the VLT, and an N-band prototype for the upcoming upgrade of the VLT mid-infrared camera VISIR (see here below).

The manufacturing process, based on the Ångström Laboratory expertise, was first presented in Ref. 15. It consists of the following steps. First, the diamond substrate is covered with a $500 \mathrm{~nm}$ aluminum coating, on top of which a mask is created into a polymer resist by electron beam lithography (e-beam). The mask pattern is then transferred into the underlying aluminum by use of a reactive ion etching process including inductively 

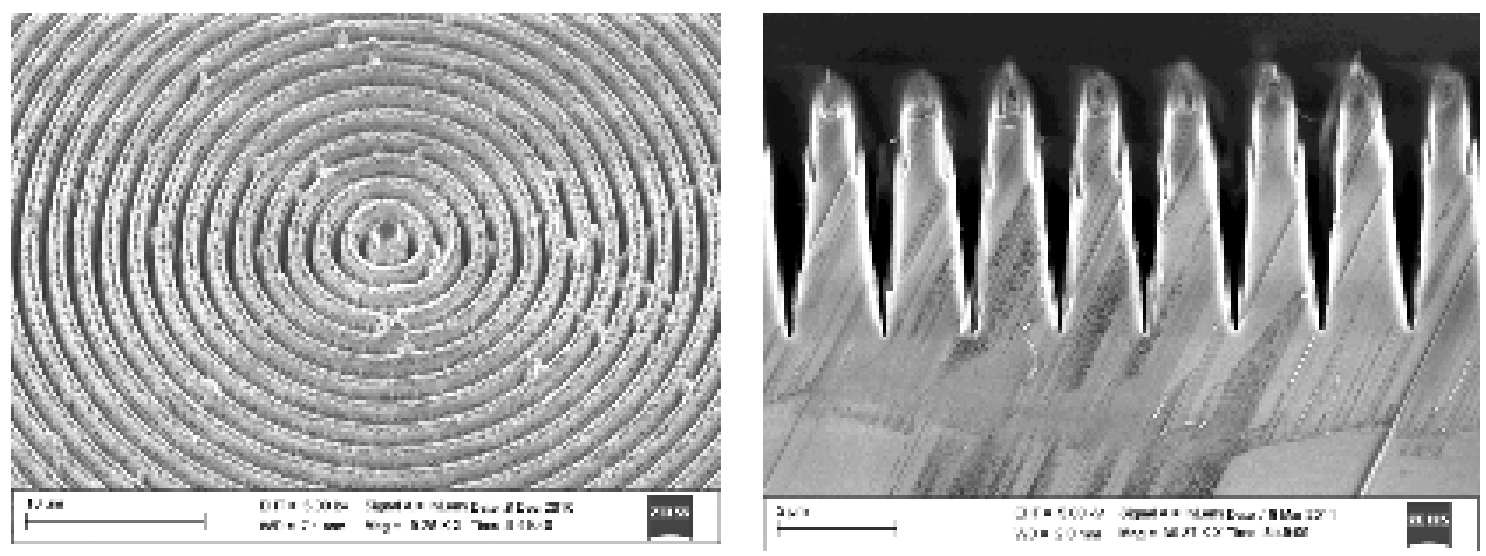

Figure 8. Left: SEM (scanning electron microscope) pictures of a topological charge 2 VVC made out of diamond subwavelength gratings, also called the Annular Groove Phase Mask (AGPM). Right: transversal cut showing the diamond subwavelength grating profile. The trapezoidal shape results from shadowing effects during reactive ion etching (the ions come from the top).

coupled plasma (RIE-ICP). The aluminum is etched in a $\mathrm{Cl}_{2} / \mathrm{BCl}_{3} / \mathrm{Ar}$ chemistry until it is etched through. The resist is then removed in acetone and the Al-mask carefully inspected by atomic force microscope (AFM) and white light interferometry. Finally, the Al-mask pattern is transferred into the diamond substrate by RIE-ICP in a $\mathrm{O}_{2} / \mathrm{Ar}$ chemistry, and the remaining aluminum is removed with solvent.

The first generation of devices (see the SEM pictures in Fig. 8) fell short of the specifications (the grating thickness was $10 \%$ off). Even if going to longer wavelengths definitely increases the feasibility of these very challenging structures, subwavelength grating tolerancing is still very tight. For instance, for an L-band device $(\lambda \approx 4 \mu \mathrm{m})$, the grating structure (thickness and feature line) needs to be controlled at the $5 \%$ level, i.e. $\sim 200$ $\mathrm{nm}$ for the thickness and $\sim 50 \mathrm{~nm}$ for the feature line. Since this first prototype, the manufacturing process has been significantly improved and the delivery of new devices is expected soon.

\subsection{Visible VVC made out of photonics crystals}

Following the successful experience of the 8-octant phase-mask coronagraph made out of photonics crystal $^{24}$ a VVC was made and tested at the Hokkaido University.

The manufacturing process (Photonic Lattice Inc.), already presented in Ref. 24 goes as follows. A periodic corrugated pattern (subwavelength grating) is precisely formed on a $1 \mathrm{~mm}$-thick substrate (synthetic fused silica) by means of electron-beam lithography with an accuracy of about $10 \mathrm{~nm}$. Two dielectric materials $\left(\mathrm{Nb}_{2} \mathrm{O}_{5}\right.$ and $\mathrm{SiO}_{2}$ ) are alternately deposited on the substrate by RF bias sputtering process preserving the corrugated surface of the substrate. The photonics-crystal VVC for visible wavelength is typically composed of about 130 layers with a thickness of about 5 microns. ${ }^{24}$ The particularity of this device is the tremendous accuracy of the center definition, believed to be sub-micrometric (Fig. 9, middle).

Preliminary results ${ }^{25}$ obtained at the Hokkaido University with active filtering of the leakage term (see Sect. 6) but without wavefront control showed speckle-limited contrast levels of the order of $\sim 10^{-6}$ at two distinct wavelengths (532 and $633 \mathrm{~nm}$ ), demonstrating the validity of the chromatic leakage filtering method exposed here below.

\section{BROADBAND OPERATIONS WITH THE VVC}

Broadband operation is the toughest challenge of phase-mask coronagraphs, and a reason why they have not been considered seriously in the most demanding high contrast imaging instrument projects so far, for a very good reason. Here we show that the VVC's vectorial, i.e. polarization properties allow for two efficient solutions that solve the chromatic dependence of this particular phase-mask coronagraph's performances. Indeed, the phase modification introduced by the VVC is geometrical, i.e. induced only by the space variation of the optical 

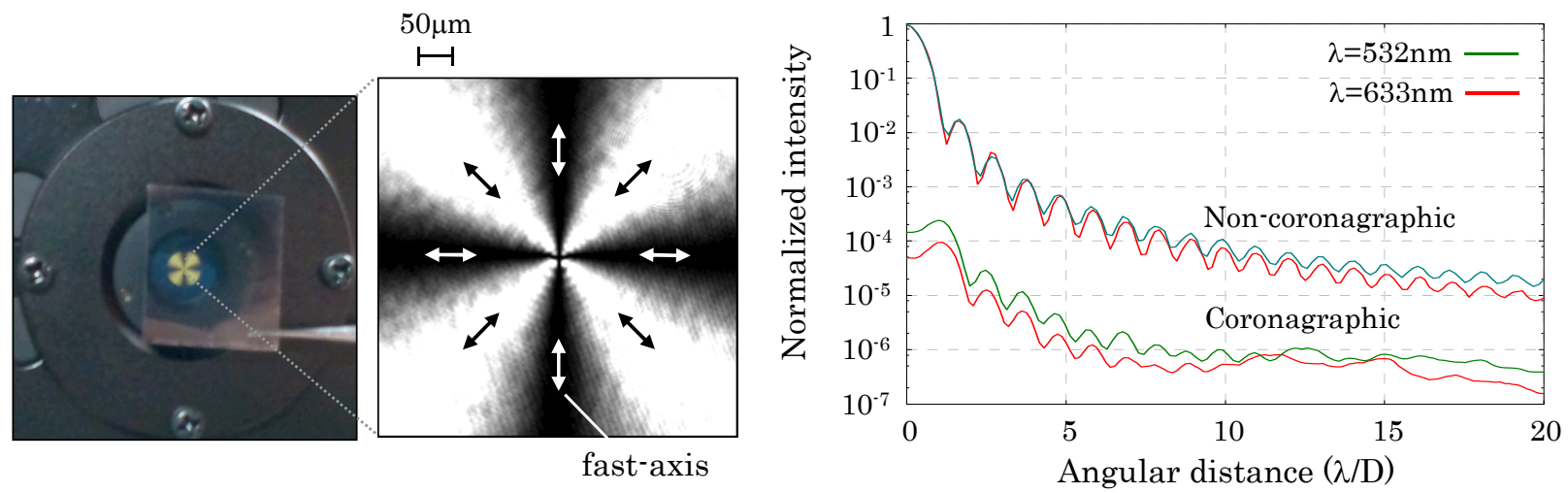

Figure 9. Left: picture of the photonics crystal VVC made in Japan (Photonics Lattice Inc.), between crossed polarizers. Middle: zoom on the central part of the PC-VVC, showing the spectacular sub-micrometric definition of the center. Right: dual-wavelength results obtained at the Hokkaido University, demonstrating the chromatic leakage filtering concept.

axis orientation across the component. Consequently, it is achromatic by nature. However, any departure from the HWP condition, as a function of wavelength for instance, makes this process lose efficiency quadratically with the retardance error. The residual is a spatially uniform chromatic leakage in the pupil, bearing no phase nor amplitude modification.

\subsection{Chromatic leakage filtering}

A rigorous analytical treatment of the VVC phase modification using the Jones formalism, ${ }^{1,6,25}$ shows that the chromatic leakage term polarization is orthogonal to the main term bearing the pure geometrical phase modification. This astonishing property suggests a radical solution to broadband operations. The polarization structure of both terms being orthogonal means that we can in principle easily get rid of the leakage term by inserting of pair of polarizer/analyzer upstream and downstream from the VVC, respectively. Indeed, an input left (resp. right) circular polarization state yields a right (resp. left) - circularly polarized vortex term and a left (resp. right) - circularly polarized leakage. An output right (resp. left) - circular analyzer would let the pure vortex term out only, blocking the left (resp. right) - circularly polarized leakage. Off-axis objects (companions, extended structures) are of course not filtered.

This solution is available to all three technological approaches to render the VVC, potentially alleviating at the same time the manufacturing constraints. Of course, the optical system receiving the VVC needs to allow additional optical elements. But in theory the gain above nominal performance level can be as high as the extinction ratio of the polarizer permits, i.e. typically $10^{4}-10^{6}$. As already mentioned, the filtering technique has been experimentally validated with a photonics crystal device. ${ }^{24,25}$ In Ref. 30 (these proceedings), which present the most recent results of the LCP VVC on the high contrast imaging testbed at JPL, we demonstrate the power and potential of this technique with the VVC made out of LCP.

\subsection{Intrinsic achromaticity}

The vectorial nature of the VVC allows intrinsically achromatic designs as well. The achromatic design methodology again makes use of the geometrical phase, but in a vertical multi-layer implementation rather than a lateral one. This principle has been known for 50 years and is even commercially available from waveplate vendors. The 3-layer VVC consists of identical retarder layers having nominal halfwave retardance values at a given unique wavelength $\lambda$. The respective optical axes are mutually offset following simple geometrical rules so as to reach an enhanced achromatic retardance across a given wavelength range. ${ }^{6}$ The 3 -layer design allows reaching the $10^{-9}$ contrast level over $20 \%$ bandwidth in theory. Note that a 5 -layer design allows reaching $10^{-12}$ over the same bandwidth (see Fig. 10, left).

In a recent attempt, JDSU manufactured a 3-layer VVC prototype made out of LCP, piling up three identical topological charge $4 \mathrm{VVC}$ satisfying the $\mathrm{HWP}$ condition around $800 \mathrm{~nm}$, with nominal offsets of their respective 

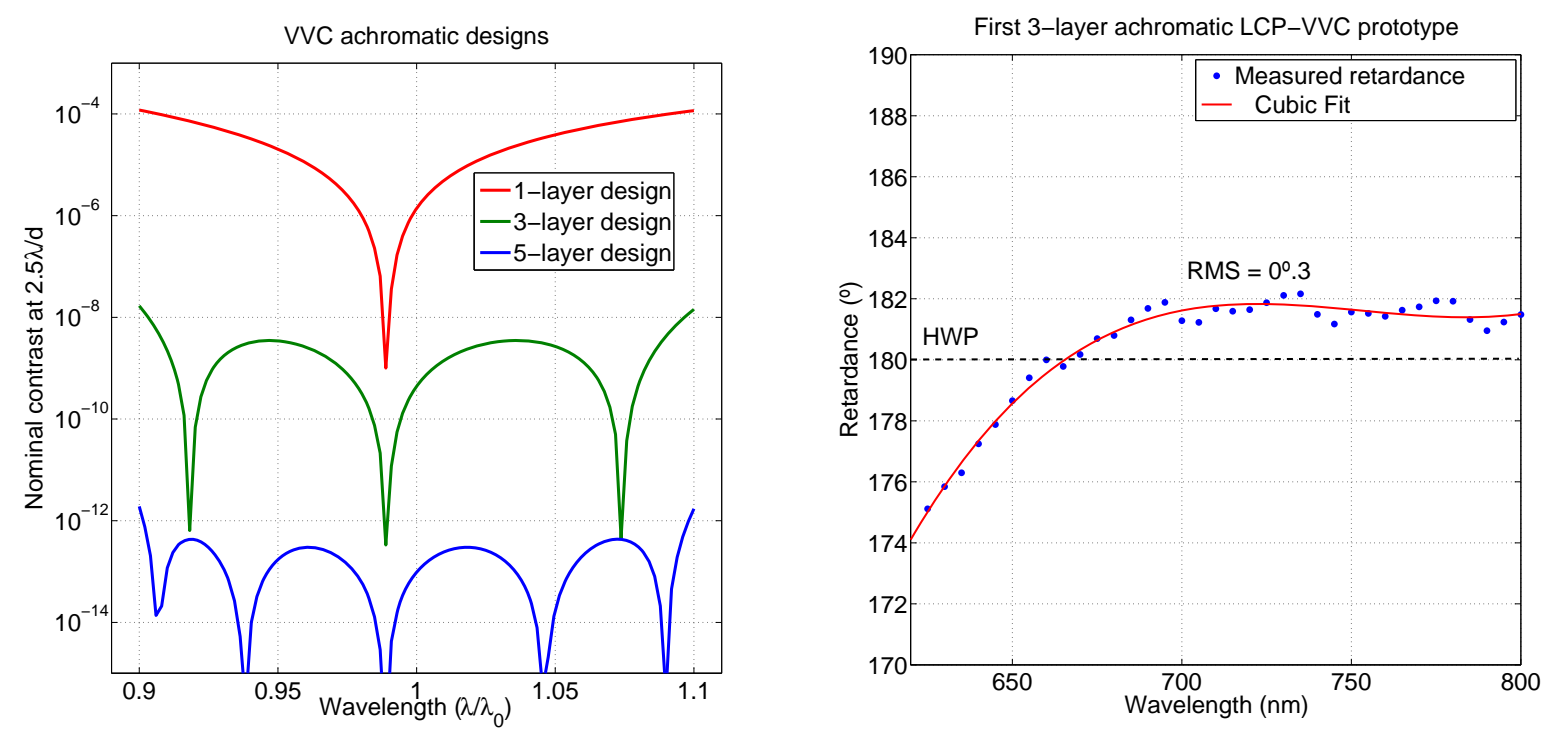

Figure 10. Left: multi-layer achromatic VVC designs. Right: 3-layer prototype made by JDSU out of LCP under contract with NASA-JPL. The measured retardance achromaticity over the 650 to $800+\mathrm{nm}$ wavelength range is such that a contrast of a few $10^{-9}$ would theoretically be possible with this device provided that the registration of the layer could be done with a micrometric accuracy as routinely done with photomask aligners in the micro-electronics industry.).

FA satisfying the achromatic 3-layer design requirement. Despite the lack of any registration mechanism, which made the central region defect as large as a couple hundred $\mu \mathrm{m}$ (and therefore the device unusable as a coronagraph), the measured retardance (Fig. 10, right) far from the center (a couple centimeters) is very close to the specification for $10^{-9}$ contrast over the $650 \mathrm{~nm}$ to $800+\mathrm{nm}$ wavelength range $(800 \mathrm{~nm}$ was the top wavelength measurable by the polarimeter, it is expected that the achromaticity extends further towards the red).

\section{PERSPECTIVES}

The VVC has always carried the promise of a close-to-ideal coronagraph: high contrast and throughput, $360^{\circ}$ discovery space, simple layout. We showed here that technologies have sufficiently matured to provide a solid ground to these claims. We will now discuss what facility can benefit from the VVC, and which of the three described technologies can meet the needs of a specific niche of the wide field of high contrast imaging.

Table 2. Qualitative (-o+) tentative comparison of the three main technologies currently being used to render the VVC according to the following criteria: manufacturability/feasibility, intrinsic achromaticity (without filtering), achromaticity (with filtering), definition of the very center of the vortex, feasibility of higher topological charges $l$ ( 4 and above), field of view or clear aperture, durability, and finally cost per device (after development cost).

\begin{tabular}{|c|c|c|c|c|c|c|c|c|}
\hline Technology & Manuf. & Achr. (w/o filt.) & Achr. (w filt.) & Center def. & Higher $l$ & FOV & Dur. & Cost \\
\hline LCP & + & + & ++ & 0 & ++ & ++ & 0 & + \\
\hline SG & - & $\mathrm{o}$ & + & ++ & - & - & ++ & - \\
\hline PC & $\mathrm{o}$ & - & + & ++ & - & - & + & - \\
\hline
\end{tabular}

\subsection{Ground-based 10-meter class telescopes}

The LCP-VVCs have already been implemented at Palomar and used in the near-infrared to image close binaries, brown dwarfs and exoplanets. ${ }^{5,28,29}$ The Palomar AO system is currently being upgraded to Palm- $3000,{ }^{32}$ the 
densest ExAO system of the foreseeable future $(<10 \mathrm{~cm}$ inter-actuator spacing). The current LCP-VVCs (optimized for $K_{s}$ and $H$ bands) or slightly enhanced versions of the same are available for use on the full superbly-corrected 5.1-meter Hale telescope aperture.

Owing to its simplicity and Lyot-like layout needs, the VVC (either the LCP-VVC or the PC-VVC) can be inserted in the coronagraphic wheel of future ExAO projects such as SPHERE, ${ }^{13}$ and HiCIAO. ${ }^{33}$ The case of $\mathrm{GPI}^{34}$ is slightly more difficult since the technical choice favored by this facility is a reflective design for the coronagraph, while each one of the technologies presented here are for transmissive coronagraphs.

Mid-infrared high contrast imaging is a wide-open field where coronagraphic devices have not been particularly appreciated so far. It has indeed been a subject of debate whether coronagraphs might or might not be needed in the thermal infrared where stellar photon noise is not the dominant source of noise, but thermal background is. However for bright targets, which in fact constitute the bulk of the exoplanet search samples, a coronagraph allows getting down to the background limited regime much closer in, especially if the coronagraph allows access to small inner working angles (IWA).

In particular, the atmospheric window ranging from 3.5 to $4.1 \mu \mathrm{m}$ (L band) is a very interesting spectral region to perform direct imaging of extrasolar planets. It actually provides a sweet spot between the best angular resolution (short wavelengths) and the best image quality (long wavelengths), while the background emission of the telescope and optical train remains moderate. The L band has indeed been successful in the past, with the discovery of the planetary companion to $\beta$ Pictoris ${ }^{35}$ and good sensitivity limits demonstrated. ${ }^{36}$

For these reasons, it will be beneficial to introduce coronagraphs to mid-infrared imagers. The SG-VVC (AGPM) is particularly adapted to this wavelength regime, and is even currently the only possible technical solution. There are two avenues currently being investigated for a VVC implementation: NAOS-CONICA, provided that the facility survives the second generation of instruments coming to the VLT, and VISIR, the mid-infrared spectro-imager of the VLT. Other mid-infrared-ready facilities are also being considered: the LargeBinocular Telescope, Keck-NIRC2, etc.

\subsection{Ground-based Extremely Large Telescopes}

The next generation of ground-based telescopes planned for the 2020 horizon will see behemoths of $30+$ meters. The main challenge will be to adaptively correct these immense apertures to get to the diffraction limit they can theoretically provide. With 10-mas class resolution in the near-infrared and unprecedented sensitivity, high contrast imagers and exoplanet science is one of the selling points of these ambitious projects. ${ }^{37,38}$ VVCs are good candidate coronagraphs for near-infrared and mid-infrared ELT instruments as shown recently. ${ }^{6,7,39}$ As in the 10-meter class telescope case, the LCP-VVC and PC-VVC are mostly adapted to short wavelength up to the near-infrared and SG-VVC are easier to manufacture for longer wavelengths.

Note that, as with most coronagraphs, centrally-obscured on-axis telescopes degrade contrast, which is especially of concern for ELTs. However, based on the remarkable ability of vortex coronagraphs to move light between the interior and exterior of pupils, we recently proposed a method, based on multiple vortices, that, without sacrificing throughput, reduces the residual light leakage to $(a / A)^{n}$, with $n \geq 4$, and $a$ and $A$ being the radii of the central obscuration and primary mirror, respectively. ${ }^{7}$ This method thus enables high contrasts to be reached even with on-axis telescopes with large central obscurations. It is worth mentioning the excellent results of a recent experiment conducted with the multi-stage four-quadrant phase-mask coronagraph and a realistic instance of an ELT entrance pupil, presented in Ref. 39.

\subsection{Space-based telescopes}

The VVC family is ideal for a space-based telescope dedicated to exoplanet imaging and characterization, as it satisfies most of the needs called for by a space-based platform: ${ }^{40}$ small IWA allowing a substantial reduction in aperture size, throughput, simple layout, lightweight. Two mission concepts are considering the VVC as a viable coronagraphic solution: ACCESS, a NASA mission concept study of an Actively Corrected Coronagraph for Exoplanet System Studies, ${ }^{41}$ and SPICES, an ESA Cosmic Vision 2 proposal for a Spectro-Polarimetric Imaging and Characterization of Exoplanetary Systems mission (Boccaletti et al. 2011, in preparation, see also 
Ref. 42,43$)$. As mentioned here above, it has also been shown recently that a tandem vortex can accommodate an on-axis telescope and still provide high contrast. ${ }^{7,44}$

Space-based projects demand the highest quality in terms of contrast provided versus bandwidth. For that, intrinsically achromatic devices will be needed. The chromatic leakage filtering option is still available, but to get down to the $10^{-11}$ contrast levels needed to characterize Earth-like planets, a combination of intrinsic and filtered achromaticity will presumably be needed. Each of the three technologies presented in this paper is potentially able to provide the needed performance. Spatialization has not been evaluated for the different technologies, but it is expected that subwavelength gratings here, owing to their monolithic nature, provide the most durable devices. However, the LCP-VVC is by far the most advanced in many areas (demonstrated contrast vs achromaticity, intrinsic and filtered), and our Palomar/HCIT experience proved the LCP-VVC can survive both vacuum and nitrogen temperatures for several years.

\section{CONCLUSION}

This paper is a snapshot of the current state of the art of three different technologies currently used to render the family of VVCs. Liquid Crystal Polymers, subwavelength gratings and photonics crystals are nowadays all mature enough to make usable devices for different applications and wavelength ranges from the optical to the mid-infrared, complementing each other instead of competitively overlapping. While some technological developments still need to be done to reach the most demanding specs of a space-based coronagraph, the general conclusion of this paper is that the portfolio of VVC manufacturing technologies is ready to provide practical answers to any kind of coronagraphic demands.

\section{ACKNOWLEDGMENTS}

This work was carried out at the European Southern Observatory (ESO) site of Vitacura (Santiago, Chile), and the Jet Propulsion Laboratory (JPL), California Institute of Technology (Caltech), under contract with the National Aeronautics and Space Administration (NASA).

\section{REFERENCES}

[1] Mawet, D., Riaud, P., Absil, O., and Surdej, J., "Annular Groove Phase Mask Coronagraph," ApJ 633, 1191-1200 (Nov. 2005).

[2] Foo, G., Palacios, D. M., and Swartzlander, Jr., G. A., "Optical vortex coronagraph," Optics Letters 30, 3308-3310 (Dec. 2005).

[3] Jenkins, C., "Optical vortex coronagraphs on ground-based telescopes," MNRAS 384, 515-524 (Feb. 2008).

[4] Swartzlander, Jr., G. A., "The optical vortex coronagraph," Journal of Optics A: Pure and Applied Optics 11, 094022-+ (Sept. 2009).

[5] Mawet, D., Serabyn, E., Liewer, K., Burruss, R., Hickey, J., and Shemo, D., "The Vector Vortex Coronagraph: Laboratory Results and First Light at Palomar Observatory," ApJ 709, 53-57 (Jan. 2010).

[6] Mawet, D., Pueyo, L., Moody, D., Krist, J., and Serabyn, E., "The Vector Vortex Coronagraph: sensitivity to central obscuration, low-order aberrations, chromaticism, and polarization," in [Society of Photo-Optical Instrumentation Engineers (SPIE) Conference Series], Society of Photo-Optical Instrumentation Engineers (SPIE) Conference Series $\mathbf{7 7 3 9}$ (July 2010).

[7] Mawet, D., Serabyn, E., Wallace, J. K., and Pueyo, L., "Improved high-contrast imaging with on-axis telescopes using a multistage vortex coronagraph," Optics Letters 36, 1506-+ (Apr. 2011).

[8] Mawet, D., Serabyn, E., Liewer, K., Hanot, C., McEldowney, S., Shemo, D., and O'Brien, N., "Optical Vectorial Vortex Coronagraphs using Liquid Crystal Polymers: theory, manufacturing and laboratory demonstration," Optics Express 17, 1902-1918 (Feb. 2009).

[9] Pancharatnam, S. Proc. Indian Acad. Sci. 44, 247 (Jan. 1956).

[10] Berry, M., "The Adiabatic Phase and Pancharatnam's Phase for Polarized Light," Journal of Modern Optics 34, 1401-1407 (Nov. 1987). 
[11] Mawet, D., Riaud, P., Baudrand, J., Baudoz, P., Boccaletti, A., Dupuis, O., and Rouan, D., "The fourquadrant phase-mask coronagraph: white light laboratory results with an achromatic device," $A \& A 448$, 801-808 (Mar. 2006).

[12] Boccaletti, A., Abe, L., Baudrand, J., Daban, J.-B., Douet, R., Guerri, G., Robbe-Dubois, S., Bendjoya, P., Dohlen, K., and Mawet, D., "Prototyping coronagraphs for exoplanet characterization with SPHERE," ArXiv e-prints $\mathbf{8 0 7}$ (July 2008).

[13] Beuzit, J.-L., Feldt, M., Dohlen, K., Mouillet, D., Puget, P., Antichi, J., Baudoz, P., Boccaletti, A., Carbillet, M., Charton, J., Claudi, R., Fusco, T., Gratton, R., Henning, T., Hubin, N., Joos, F., Kasper, M., Langlois, M., Moutou, C., Pragt, J., Rabou, P., Saisse, M., Schmid, H. M., Turatto, M., Udry, S., Vakili, F., Waters, R., and Wildi, F., "SPHERE: A Planet Finder Instrument for the VLT," in [In the Spirit of Bernard Lyot: The Direct Detection of Planets and Circumstellar Disks in the 21st Century], Kalas, P., ed. (June 2007).

[14] McKenzie, P. A., Hendrix, K., Shemo, D., and Tan, K., "Hybrid optical retarders fabricated from liquid crystal polymer and form birefringent thin films," in [Society of Photo-Optical Instrumentation Engineers (SPIE) Conference Series], Society of Photo-Optical Instrumentation Engineers (SPIE) Conference Series 6972 (May 2008).

[15] Karlsson, M. and Nikolajeff, F., "Diamond micro-optics: microlenses and antireflection structured surfaces for the infrared spectral region," Optics Express 11, 502-+ (Mar. 2003).

[16] Niv, A., Biener, G., Kleiner, V., and Hasman, E., "Polychromatic vectorial vortex formed by geometric phase elements," Optics Letters 32, 847-849 (Apr. 2007).

[17] McEldowney, S. C., Shemo, D. M., Chipman, R. A., and Smith, P. K., "Creating vortex retarders using photoaligned liquid crystal polymers," Optics Letters 33, 134-+ (2008).

[18] McEldowney, S. C., Shemo, D. M., and Chipman, R. A., "Vortex retarders produced from photo-aligned liquid crystal polymers," Optics Express 16, 7295-+ (May 2008).

[19] Kikuta, H., Ohira, Y., and Iwata, K., "Achromatic quarter-wave plates using the dispersion of form birefringence," Appl. Opt. 36, 1566-1572 (Mar. 1997).

[20] Deguzman, P. C. and Nordin, G. P., "Stacked Subwavelength Gratings as Circular Polarization Filters," Appl. Opt. 40, 5731-5737 (Nov. 2001).

[21] Mawet, D., Riaud, P., Surdej, J., and Baudrand, J., "Subwavelength surface-relief gratings for stellar coronagraphy," Appl. Opt. 44, 7313-7321 (Dec. 2005).

[22] Deng, X., Liu, F., Wang, J. J., Sciortino, Jr., P. F., Chen, L., and Liu, X., "Achromatic wave plates for optical pickup units fabricated by use of imprint lithography," Optics Letters 30, 2614-2616 (Oct. 2005).

[23] Murakami, N., Uemura, R., Baba, N., Nishikawa, J., Tamura, M., Hashimoto, N., and Abe, L., "An EightOctant Phase-Mask Coronagraph," PASP 120, 1112-1118 (Oct. 2008).

[24] Murakami, N., Nishikawa, J., Yokochi, K., Tamura, M., Baba, N., and Abe, L., "Achromatic Eight-octant Phase-mask Coronagraph using Photonic Crystal," ApJ 714, 772-777 (May 2010).

[25] Murakami, N., Baba, N., Ise, A., Sakamoto, M., and Oka, K., "Laboratory demonstration of an optical vortex mask coronagraph using photonic crystal," in [In the Spirit of Lyot 2010], (Oct. 2010).

[26] Kawakami, S., Kawashima, T., and Sato, T., "Mechanism of shape formation of three-dimensional periodic nanostructures by bias sputtering," Applied Physics Letters 74, 463-465 (Jan. 1999).

[27] Kawashima, T., Miura, K., Sato, T., and Kawakami, S., "Self-healing effects in the fabrication process of photonic crystals," Applied Physics Letters 77, 2613-2615 (Oct. 2000).

[28] Mawet, D., Mennesson, B., Serabyn, E., Stapelfeldt, K., and Absil, O., "A dim candidate companion to $\epsilon$ Cephei," ArXiv e-prints (July 2011).

[29] Serabyn, E., Mawet, D., and Burruss, R., "An image of an exoplanet separated by two diffraction beamwidths from a star," Nature 464, 1018-1020 (Apr. 2010).

[30] Mawet, D., Serabyn, E., Moody, D., Kern, B., Niessner, E., Kuhnert, A., and Trauger, J., "Recent results of the second generation of vector vortex coronagraphs on the high-contrast imaging testbed at JPL," in [Society of Photo-Optical Instrumentation Engineers (SPIE) Conference Series], Society of Photo-Optical Instrumentation Engineers (SPIE) Conference Series (2011).

[31] Nersisyan, S. R., Tabiryan, N. V., Steeves, D. M., and Kimball, B. R., "The Promise of Diffractive Waveplates," Optics $\& 3$ Photonics News 21, 40-+ (Mar. 2010). 
[32] Dekany, R., Bouchez, A., Britton, M., Velur, V., Troy, M., Shelton, J. C., and Roberts, J., "PALM-3000: visible light AO on the 5.1-meter Telescope," in [Advances in Adaptive Optics II. Edited by Ellerbroek, Brent L.; Bonaccini Calia, Domenico. Proceedings of the SPIE, Volume 6272, pp. 62720G (2006).], Presented at the Society of Photo-Optical Instrumentation Engineers (SPIE) Conference 6272 (July 2006).

[33] Tamura, M., Hodapp, K., Takami, H., Abe, L., Suto, H., Guyon, O., Jacobson, S., Kandori, R., Morino, J.-I., Murakami, N., Stahlberger, V., Suzuki, R., Tavrov, A., Yamada, H., Nishikawa, J., Ukita, N., Hashimoto, J., Izumiura, H., Hayashi, M., Nakajima, T., and Nishimura, T., "Concept and science of HiCIAO: high contrast instrument for the Subaru next generation adaptive optics," in [Ground-based and Airborne Instrumentation for Astronomy. Edited by McLean, Ian S.; Iye, Masanori. Proceedings of the SPIE, Volume 6269, pp. 62690V (2006).], Presented at the Society of Photo-Optical Instrumentation Engineers (SPIE) Conference 6269 (July 2006).

[34] Macintosh, B., Graham, J., Palmer, D., Doyon, R., Gavel, D., Larkin, J., Oppenheimer, B., Saddlemyer, L., Wallace, J. K., Bauman, B., Erikson, D., Poyneer, L., Sivaramakrishnan, A., Soummer, R., and Veran, J.-P., "Adaptive optics for direct detection of extrasolar planets: the Gemini Planet Imager," Comptes Rendus Physique 8, 365-373 (Apr. 2007).

[35] Lagrange, A.-M., Gratadour, D., Chauvin, G., Fusco, T., Ehrenreich, D., Mouillet, D., Rousset, G., Rouan, D., Allard, F., Gendron, É., Charton, J., Mugnier, L., Rabou, P., Montri, J., and Lacombe, F., "A probable giant planet imaged in the $\beta$ Pictoris disk. VLT/NaCo deep L'-band imaging," $A \& A 493$, L21-L25 (Jan. 2009).

[36] Kasper, M., Apai, D., Janson, M., and Brandner, W., "A novel L-band imaging search for giant planets in the Tucana and $\beta$ Pictoris moving groups," $A \& A A$ 472, 321-327 (Sept. 2007).

[37] Kasper, M., Beuzit, J.-L., Verinaud, C., Gratton, R. G., Kerber, F., Yaitskova, N., Boccaletti, A., Thatte, N., Schmid, H. M., Keller, C., Baudoz, P., Abe, L., Aller-Carpentier, E., Antichi, J., Bonavita, M., Dohlen, K., Fedrigo, E., Hanenburg, H., Hubin, N., Jager, R., Korkiakoski, V., Martinez, P., Mesa, D., Preis, O., Rabou, P., Roelfsema, R., Salter, G., Tecza, M., and Venema, L., "EPICS: direct imaging of exoplanets with the E-ELT," in [Society of Photo-Optical Instrumentation Engineers (SPIE) Conference Series], Society of Photo-Optical Instrumentation Engineers (SPIE) Conference Series 7735 (July 2010).

[38] Brandl, B. R., Lenzen, R., Pantin, E., Glasse, A., Blommaert, J., Venema, L., Molster, F., Siebenmorgen, R., Kendrew, S., Baes, M., Böhnhardt, H., Brandner, W., van Dishoeck, E., Henning, T., Käufl, H. U., Lagage, P.-O., Moore, T. J. T., Waelkens, C., and van der Werf, P., "Instrument concept and science case for the mid-IR E-ELT imager and spectrograph METIS," in [Society of Photo-Optical Instrumentation Engineers (SPIE) Conference Series], Society of Photo-Optical Instrumentation Engineers (SPIE) Conference Series 7735 (July 2010).

[39] Galicher, R., Baudoz, P., and Baudrand, J., "Multi-stage four-quadrant phase mask: achromatic coronagraph for space-based and ground-based telescopes," A\& A 530, A43+ (June 2011).

[40] Serabyn, E., Mawet, D., and Wallace, J., "Recent progress in vector vortex coronagraphy ," in [Society of Photo-Optical Instrumentation Engineers (SPIE) Conference Series], Society of Photo-Optical Instrumentation Engineers (SPIE) Conference Series (2011).

[41] Trauger, J., Stapelfeldt, K., Traub, W., Henry, C., Krist, J., Mawet, D., Moody, D., Park, P., Pueyo, L., Serabyn, E., Shaklan, S., Guyon, O., Kasdin, J., Spergel, D., Vanderbei, R., Belikov, R., Marcy, G., Brown, R. A., Schneider, J., Woodgate, B., Matthews, G., Egerman, R., Polidan, R., Lillie, C., Ealey, M., and Price, T., "ACCESS: a NASA mission concept study of an Actively Corrected Coronagraph for Exoplanet System Studies," in [Society of Photo-Optical Instrumentation Engineers (SPIE) Conference Series], Society of Photo-Optical Instrumentation Engineers (SPIE) Conference Series 7010 (Aug. 2008).

[42] Maire, A.-L., Galicher, R., Boccaletti, A., Schneider, J., and Baudoz, P., "Visible spectroscopy of terrestrial exoplanets with SEE-COAST," in [SF2A-2010: Proceedings of the Annual meeting of the French Society of Astronomy and Astrophysics], S. Boissier, M. Heydari-Malayeri, R. Samadi, \& D. Valls-Gabaud, ed., 81-+ (Dec. 2010).

[43] Boccaletti, A., Sozzetti, A., Schneider, J., Baudoz, P., Tinetti, G., and Stam, D., "The SEE-COAST concept," Highlights of Astronomy 15, 718-719 (Nov. 2010).

[44] Serabyn, E., Wallace, J., and Mawet, D., "Speckle phase measurement in a tandem vortex coronagraph," (2011). 